\title{
Editorial: special issue on time series extremes
}

\author{
Rafal Kulik ${ }^{1}$
}

Received: 14 June 2016 / Accepted: 15 June 2016 /

Published online: 7 July 2016

(C) Springer Science+Business Media New York 2016

Extreme value theory (EVT) provides an asymptotically justified framework for the statistical modeling of rare events. For independent, identically distributed (i.i.d.) univariate random variables or random vectors, the theory dates back to the 1920s and the celebrated Fisher-Tippett-Gnedenko theorem that describes possible limits of normalized maxima. The limiting distributions are called Weibull, Gumbel or Fréchet. The Fréchet distribution has regularly varying (i.e., power-law tail with a positive index $\alpha$ ). This distribution and its maximum domain of attraction are suitable models for describing heavy-tail phenomena. Regular variation also provides a link between EVT and powerful probabilistic tools such as point processes and their weak convergence. The theoretical foundations triggered the development of suitable statistical methods, including the Peaks Over Threshold (POT) approach and Generalized Pareto Distribution (GPD) fitting. In the Fréchet domain case, different estimators of the tail index, including the celebrated Hill estimator, were studied. Although some statistical challenges remain, one can argue that EVT for i.i.d. random variables and vectors is well understood and summarized in research monographs like (Embrechts et al. 1997) or (de Haan and Ferreira 2006).

However, the situation is more complicated when dealing with dependent (stationary or non-stationary) or complex (such as multivariate or function-valued) data. In this special issue we focus on stationary time series $\left\{X_{j}, j \geq 0\right\}$ only. For such random processes, standard procedures use marginal extreme value modeling (the same as in the i.i.d. case mentioned before), but consideration of the serial dependence structure between the variables is essential, for example, when assessing risk due to clusters of extremes. In (Leadbetter et al. 1983) and (O'Brien 1987) the authors provided distinct characterisations of clustering through the extremal index $\theta \in[0,1]$.

Rafal Kulik

rkulik@uottawa.ca

1 University of Ottawa, Ottawa, Canada 
If $\theta \in(0,1)$, then the limiting distribution of the maxima of a stationary time series is again of the Weibull, Gumbel or Fréchet-type, properly re-parametrized to incorporate the extremal index. If $\theta=1$, then maxima behave asymptotically as if the random variables $X_{j}$ were independent, like in the case of any Gaussian process whose correlation function decays faster than logarithmically. This example also indicates that serial and extremal dependence are two different concepts. The clustering of extremes appears also in the aforementioned point process methodology, where the typical limit for weakly dependent time series is a cluster Poisson process; see for example (Hsing et al. 1988; Davis and Hsing 1995) and (Basrak et al. 2012).

Statistical methodology for extremes of stationary time series seems less developed. The most popular approach is the block method. The observations $X_{1}, \ldots, X_{n}$ from a stationary time series are divided into $m_{n}$ non-overlapping blocks of size $r_{n}$. Each block is treated as one cluster. The extremal index is estimated by calculating the ratio of the number of blocks with at least one value exceeding a high threshold and the number of exceedances over the threshold in the entire set of observations; see (Hsing 1991a; Smith and Weissman 1994). As argued in (Smith and Weissman 1994), the block estimator of the extremal index is a natural starting point for any statistical study related to time series extremes. In particular, the distribution of $M_{n}=\max \left\{X_{1}, \ldots, X_{n}\right\}$ is approximated as

$$
\mathbb{P}\left(M_{n} \leq x\right) \approx \mathbb{P}^{n \theta}\left(X_{0} \leq x\right)
$$

Another statistical problem of interest is estimation of the tail index in case of regularly varying time series. In (Hsing 1991b) the author develops the limiting theory for a general class of stationary processes, while some additional ad-hoc approaches exist for specific time series models, such as linear processes; see (Resnick and Stărică 1998).

In order to obtain a general statistical theory for stationary time series, Rootzén et al. (1998) consider tail array sums and their asymptotic normality under mixing conditions. This line of work has been continued by exploiting techniques for the tail empirical processes, culminating in Drees and Rootzén (2010).

Many challenges remain, both from a probabilistic and a statistical perspective, including:

(a) How to measure extremal dependence in univariate and multivariate time series?

(b) Statistical inference for extremal dependence between components of multivariate time series.

(c) How to model extremal independence in time series?

(d) How to deal with high-dimensional time series?

(e) Probabilistic properties of multivariate heavy-tailed time series.

For (a) we would like to point out the recent work on the extremogram by (Davis and Mikosch 2009), while for (b) we would like to mention that the blocking method generally fails to capture the extremal dependence between the components, rather allows to estimate the dependence structure between the block-maxima; see Bücher and Segers (2014). 
In this special issue we deal with partial answers to problems (c), (d) and (e). The paper by T. McElroy (McElroy 2016) presents an extensive review of extremes for time series. Two different points of view are presented: extremes as natural part of the random mechanism (in the context of insurance, finance or extreme weather prediction); or extreme values merely as a nuisance. From the first perspective, the author starts the discussion with the above mentioned notion of extremal clustering and its link to convergence of point processes to a cluster Poisson process. Different approaches how to measure extremal dependence are discussed. Statistical methodology follows - different estimators of the extremal index and of the tail index are presented. From the second perspective, mostly for forecasting and signal extraction problems, there are two paradigms. First, one may want to remove the extreme values and then proceed with a methodology that is optimal for light-tailed time series, or one may keep the extremes in the data and instead utilize a methodology that is robust with respect to the presence of extremes. The paper is concluded with an extensive list of references - a quick guide for all who start working in the area.

T. Lugrin, A. Davison and J. Tawn (Lugrin et al. (2016)) tackle problem (c): the modeling of univariate stationary time series $\left\{X_{j}\right\}$ with extremal independence. For such time series, the extremal index is $\theta=1$. In this case, the asymptotic behavior of the maxima of a time series cannot be distinguished from the corresponding behavior of the maxima of an i.i.d. sequence with the same marginals. Furthermore, in case of extremal independence the limiting conditional probabilities $\mathbb{P}\left(X_{j}>x \mid X_{0}>x\right)$ when $x \rightarrow \infty$, vanish for $j \geq 1$ and no relevant information can be inferred. Following an idea of Ledford and Tawn (1996), the aforementioned authors and various others ((Heffernan and Resnick 2007; Kulik and Soulier 2015) to name a few), considered $\mathbb{P}\left(X_{j}>b(x) \mid X_{0}>x\right)$, where $b(x)$ grows slower than $x$. The authors of the presented paper discuss different approaches for modeling and estimation, including the Bayesian framework.

Big data and high-dimensional phenomena are hot topics in modern statistics. Although extreme value theory is related rather to small data and rare events, highdimensionality occurs naturally in multivariate time series. One of the challenging problems is the behavior of the eigenvalues and eigenvectors of sample covariance matrices associated with multivariate time series whose dimension increases together with the sample size. R. Davis, T. Mikosch, J. Heiny, X. Xie (Davis et al. (2016)) consider multivariate linear and i.i.d. heavy-tailed time series and discuss the asymptotic behavior of the eigenvalues of such random matrices. The authors argue that the properly normalized ordered sample eigenvalues of the sample covariance matrices have distributional limits given by the points of a suitable Poisson process. In particular, the normalized largest eigenvalue converges to a Fréchet distribution, unlike the light-tailed case, where the celebrated Tracy-Widom law appears.

Finally, B. Basrak and A. Tafro (Basrak and Tafro (2016)) deal with problem (e). Limit theorems for heavy-tailed time series are a challenging problem. For example, for linear heavy-tailed time series it is known from Avram and Taqqu (1992), that the functional central limit theorem of the partial sum process generated from this time series does not hold in the Skorokhod topology $J_{1}$. This observation has led to research on alternative topologies and different ways to prove the functional convergence for some classes of weakly dependent stationary time series with heavy tails. 
The authors make another step in this direction, employing a promising technique which is based on the tail and spectral tail processes of regularly varying time series models; see Basrak and Segers (2009).

In summary, we believe that the presented four papers deal with some relevant problems related to the probabilistic and statistical aspects of extreme value theory for stationary time series. This special issue provides overviews of some of the modern areas of research. We hope the problems mentioned in this issue will stimulate novel research in the field of extremes for dependent data.

Rafał Kulik, Ottawa, June 15, 2016

\section{References}

Avram, F., Taqqu, M.S.: Weak convergence of sums of moving averages in the $\alpha$-stable domain of attraction. Ann. Probab. 20, 483-503 (1992)

Basrak, B., Krizmanić, D., Segers, J.ohan.: A functional limit theorem for dependent sequences with infinite variance stable limits. Ann. Probab. 40(5), 2008-2033 (2012)

Basrak, B., Segers, J.: Regularly varying multivariate time series. Stochastic Processes and their Applications 119(4), 1055-1080 (2009)

Basrak, B., Tafro, A.: A complete convergence theorem for stationary regularly varying multivariate time series. Extremes 20(3) (2016)

Bücher, A., Segers, J.: Extreme value copula estimation based on block maxima of a multivariate stationary time series. Extremes 17, 495-528 (2014)

Davis, R.A., Hsing, T.: Point process and partial sum convergence for weakly dependent random variables with infinite variance. Ann. Probab. 23(2), 879-917 (1995)

Davis, R.A., Mikosch, T.: The extremogram: A correlogram for extreme events. Bernoulli 38A, 977-1009 (2009). Probability, statistics and seismology

Davis, R.A., Heiny, J., Mikosch, T., Xie, X.: Extreme value analysis for the sample autocovariance matrices of heavy-tailed multivariate time series. Extremes 20(3) (2016)

de Haan, L., Ferreira, A.: Extreme value theory. Springer Series in Operations Research and Financial Engineering. Springer, New York (2006). An introduction

Drees, H., Rootzén, H.: Limit theorems for empirical processes of cluster functionals. Ann. Stat. 38(4), 2145-2186 (2010)

Embrechts, P., Klüppelberg, C., Mikosch, T.: Modelling extremal events, volume 33 of Applications of Mathematics. Springer-Verlag, Berlin (1997)

Heffernan, J.E., Resnick, S.I.: Limit laws for random vectors with an extreme component. Ann. Appl. Probab. 17(2), 537-571 (2007)

Hsing, T., Hüsler, J., Ross Leadbetter, M.: On the exceedance point process for a stationary sequence. Probab. Theory Relat. Fields 78, 97-112 (1988)

Hsing, T.: Estimating the parameters of rare events. Stochastic Processes and their Applications (1991)

Hsing, T.: On tail index estimation using dependent data. Ann. Stat. 19, 1547-1569 (1991)

Kulik, R., Soulier, P.: Heavy tailed time series with extremal independence. Extremes 18, 273-299 (2015)

Leadbetter, R.M., Lindgren, G., Rootzén, H.: Extremes and related properties of random sequences and processes. Springer Series in Statistics. Springer-Verlag, New York (1983)

Ledford, A.W., Tawn, J.A.: Statistics for near independence in multivariate extreme values. Biometrika 83(1), 169-187 (1996)

Lugrin, T., Davison, A., Tawn, J.: Bayesian uncertainty management in temporal dependence of extremes. Extremes 20(3) (2016)

McElroy, T.: On the measurement and treatment of extremes in time series. Extremes 20(3) (2016)

O'Brien, G.L.: Extreme values for stationary and Markov sequences. Ann. Probab. 15(1), 281-291 (1987)

Resnick, S., Stărică, C.: Tail index estimation for dependent data. Ann. Appl. Probab. 8(4), 1156-1183 (1998)

Rootzén, H., Leadbetter, R.M., de Haan, L.: On the distribution of tail array sums for strongly mixing stationary sequences. Ann. Appl. Probab. 8(3), 868-885 (1998)

Smith, R.L., Weissman, I.: Estimating the extremal index. J. R. Statist. Soc. B 56, 515-528 (1994) 